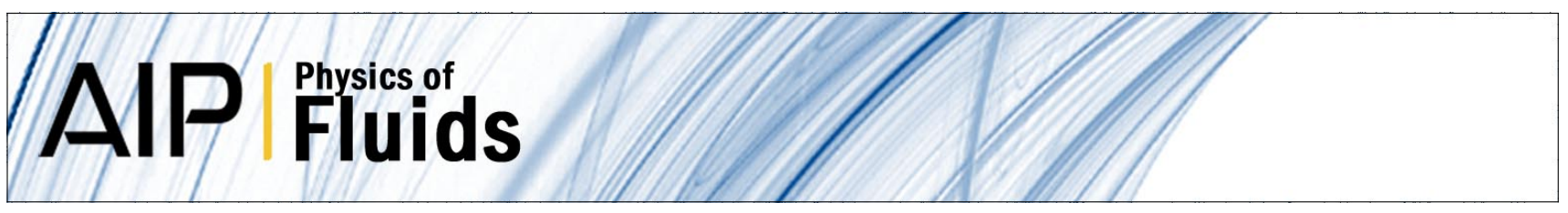

\title{
Convective Instabilities in Plane Couette Flow
}

Andrew P. Ingersoll

Citation: Phys. Fluids 9, 682 (1966); doi: 10.1063/1.1761733

View online: http://dx.doi.org/10.1063/1.1761733

View Table of Contents: http://pof.aip.org/resource/1/PFLDAS/v9/i4

Published by the American Institute of Physics.

\section{Additional information on Phys. Fluids}

Journal Homepage: http://pof.aip.org/

Journal Information: http://pof.aip.org/about/about_the_journal

Top downloads: http://pof.aip.org/features/most_downloaded

Information for Authors: http://pof.aip.org/authors

\section{ADVERTISEMENT}

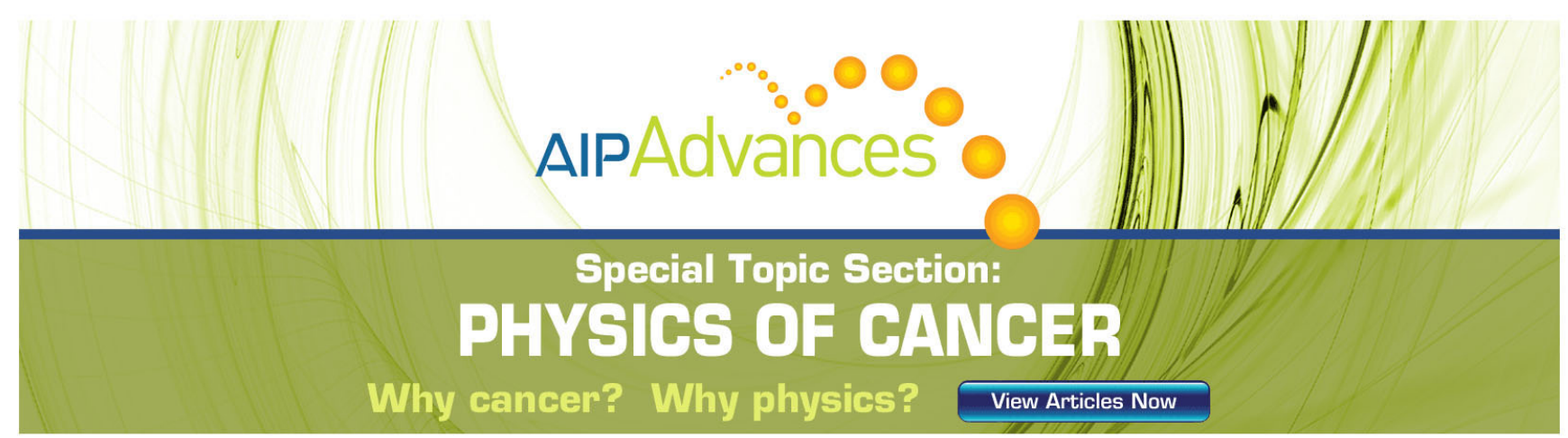




\title{
Convective Instabilities in Plane Couette Flow
}

\author{
ANDREW P. INGERSOLL \\ Harvard University, Cambridge, Massachusetts
}

(Received 8 December 1965)

\begin{abstract}
The stability to infinitesimal disturbances of plane Couette flow is considered in the presence of a negative vertical temperature gradient. The fluid is contained between horizontal planes which are maintained at different temperatures. The flow becomes unstable at the critical Rayleigh number for convection without shear. Below this critical Rayleigh number, the flow is stable in the limit of large and small Reynolds numbers. Numerical solutions at finite Reynolds numbers are given for stationary and traveling disturbances at Prandtl numbers equal to ten and infinity. For these cases, the flow appears stable at subcritical Rayleigh numbers, for all Reynolds numbers.
\end{abstract}

\section{INTRODUCTION}

$\mathbf{A}$ FLUID is contained between horizontal planes on which the temperature and velocity are fixed. These bounding surfaces move relative to each other with constant horizontal velocity, and the lower surface is maintained at a constant temperature above that of the upper surface. Experiments on air by Benard and Avsec, ${ }^{1}$ Chandra, ${ }^{2}$ and Brunt ${ }^{3}$ indicate that convection usually occurs as longitudinal rolls, with axes parallel to the mean flow. Transverse rolls are also observed at small rates of shear.

Kuo $^{4}$ has examined the stability of the basic state analytically, using a nondissipative model. More recently, Deardorff, ${ }^{5}$ Gallagher and Mercer, ${ }^{6}$ and this writer ${ }^{7}$ analyzed the full sixth-order stability problem numerically. The results were obtained independently using different methods and agree within quite accurate limits. Curves of neutral stability were calculated relating the five physical parameters of the problem. These are the Rayleigh number, the Prandtl number, the phase speed and horizontal wavenumber of the disturbance, and the Reynolds number based on the component of the mean velocity normal to the wave fronts of the disturbance. When this last parameter is zero (e.g., for longitudinal rolls), the equations reduce to those of the Rayleigh stability problem, treated by Pellew and Southwell. ${ }^{8}$ For that problem, in which there is no mean shear, unstable disturbances

\footnotetext{
${ }^{1}$ H. Benard and D. Avsec, J. Phys. Radium 9, 486 (1938).

2 K Chandra, Proc. Roy. Soc. (London) A164, 231 (1938).

${ }^{3} \mathrm{D}$. Brunt, in Compendium of Meteorology (American Meteorological Society, Boston, Massachusetts, 1951), p. 1255.

${ }^{4}$ H. L. Kuo, Phys. Fluids 6, 195 (1963).

5 J. W. Deardorff, Phys. Fluids 8, 1027 (1965).

${ }^{6}$ A. P. Gallagher and A. McD. Mercer, Proc. Roy. Soc. (London) A286, 117 (1965).

7 A. P. Ingersoll, Ph.D. thesis, Harvard University (1965)

8 A. Pellew and R. V. Southwell, Proc. Roy. Soc. (London) A176, 312 (1940).
}

will exist at values of the Rayleigh number above a certain critical value which is independent of the Prandtl number. Thus with a mean shear, longitudinal rolls will be unstable above this critical Rayleigh number, and so the basic state cannot exist in this range.

The only question of physical interest is then whether the basic state with shear is unstable at Rayleigh numbers below this critical value, for disturbances other than longitudinal rolls. Deardorff $f^{5}$ considered stationary disturbances (those moving with the fluid midway between the boundaries), at several values of the Prandtl number, for Reynolds numbers less than 300. Gallagher and Mercer ${ }^{6}$ studied a larger range of Reynolds numbers and gave an example of a neutrally stable traveling disturbance. The Rayleigh number at neutral stability seems to increase with Reynolds number and with Prandtl number, and the wavelength of the most unstable disturbance increases with the Reynolds number. This writer ${ }^{7}$ has obtained similar results for a comparable range of parameters. No cases of instability were found at Rayleigh numbers below the critical value for longitudinal rolls.

Unfortunately, these numerical solutions do not cover all cases of interest. Neutral stability curves for traveling disturbances were not obtained, and the data do not illustrate fully the effect of Prandtl number variation. Moreover, Deardorff speculates that the Rayleigh number at neutral stability might decrease for Reynolds numbers above a certain value, although no results could be obtained at very large Reynolds numbers. Below we direct our attention to these questions. We conclude, with Deardorff, and with Gallagher and Mercer, that the flow is stable below the critical Rayleigh number for longitudinal rolls. This and the preceding work do not constitute a rigorous proof of stability, however, and so our conclusion remains tentative. 


\section{MATHEMATICAL FORMULATION}

Let $\left(u^{\prime}, v^{\prime}, w^{\prime}\right)$ be the velocity and $T^{\prime}$ be the temperature of the fluid at a point $\left(x^{\prime}, y^{\prime}, z^{\prime}\right)$ at time $\ell^{\prime}$. The boundaries are taken to lie at $z^{\prime}= \pm \frac{1}{2} h$, where $z^{\prime}$ is the vertical coordinate. The basic state is then

$u^{\prime}=\Delta u z^{\prime} / h, \quad v^{\prime}=w^{\prime}=0, \quad T^{\prime}=-\Delta T z^{\prime} / h$.

Consider a disturbance of the form

$$
f\left(z^{\prime}\right) \exp \left\{\left[i\left(x^{\prime}-\Delta u c t^{\prime}\right) \alpha_{x} / h\right]+\left(i y^{\prime} \alpha_{y} / h\right)\right\} .
$$

Then let $\alpha=\left(\alpha_{x}^{2}+\alpha_{y}^{2}\right)^{\frac{1}{2}}$ be the (positive) horizontal wavenumber, scaled by the quantity $1 / h$. The real part of $c$ is the phase speed of the disturbance in the $x^{\prime}$ direction, scaled by the velocity $\Delta u$. The imaginary part is related to the growth rate and is positive for an unstable disturbance.

We assume that the fluid obeys the Boussinesq equations of motion. ${ }^{\mathbf{8} 9}$ Then let $w$ and $\theta$ be the dimensionless vertical velocity and temperature of an infinitesimal disturbance of the form (2). We may treat $w$ and $\theta$ as functions only of $z$, the dimensionless vertical coordinate, whence

$$
\begin{aligned}
{\left[\nabla^{2}-i \alpha \epsilon(z-c)\right] \nabla^{2} w } & =\alpha^{2} R \theta, \\
{\left[\nabla^{2}-i \sigma \alpha \epsilon(z-c)\right] \theta } & =-w .
\end{aligned}
$$

Here $\nabla^{2}$ is the Laplacian operator $D^{2}-\alpha^{2}$, where $D$ is the derivative with respect to $z$. $R$ is the Rayleigh number and $\sigma$ the Prandtl number,

$$
R=\gamma \Delta T h^{3} /(\nu \kappa), \quad \sigma=\nu / \kappa,
$$

where $\nu$ is the kinematic viscosity, $\kappa$ the thermometric conductivity, and $\gamma$ is the product of the gravitational acceleration and the thermal coefficient of expansion of the fluid. Finally, $\epsilon$ is the Reynolds number based on the transverse component of the basic flow,

$$
\epsilon=\operatorname{Re} \alpha_{x} / \alpha=(\Delta u h / \nu)\left(\alpha_{x} / \alpha\right) .
$$

The boundary conditions are

$$
w=D w=\theta=0 \quad\left(z= \pm \frac{1}{2}\right),
$$

corresponding to rigid, conducting plane surfaces.

The quantity $\alpha_{x} / \alpha$ in (6) is the cosine of the angle between the disturbance wave vector and the direction of the mean flow. For transverse rolls $\left[\left(\alpha_{x} / \alpha\right)=1\right], \epsilon$ is equal to Re, the Reynolds number of the basic state. For longitudinal rolls $\left[\left(\alpha_{x} / \alpha\right)=\epsilon=0\right]$, the equations are those of the Rayleigh stability problem. So at $R \approx 1708$,

${ }^{9} \mathrm{~S}$. Chandrasekhar, Hydrodynamic and Hydromagnetic Stability (Oxford University Press, Oxford, 1961), p. 16. longitudinal rolls of wavenumber $\alpha \approx 3.117$ will become unstable. We also wish to determine whether there are disturbances with $\epsilon \neq 0$ which are unstable at $R<1708$. Thus we must calculate the eigenvalues $R$ for which (3), (4), and (7) are satisfied, for all $\sigma, \alpha, \epsilon$, and $c$. We consider only curves of neutral stability ( $c$ real), and for definiteness we shall take $\sigma, \alpha, \epsilon$, and $c$ all positive. (Actually, $\pm c$ are equally valid solutions, corresponding to waves traveling with equal and opposite velocities.) In the next section we develop the means of obtaining these eigenvalues.

\section{THE EIGENFUNCTION EXPANSION}

Expand $w$ and $\theta$ in terms of normalized functions of $z$ satisfying the boundary conditions (7),

$$
w=\sum_{n=0}^{\infty} b_{n} f_{n}(z), \quad \theta=\sum_{n=0}^{\infty} d_{n} g_{n}(z),
$$

where

$$
f_{n}=D f_{n}=g_{n}=0 \quad\left(z= \pm \frac{1}{2}\right) .
$$

In the present problem, it is useful to determine these functions by requiring that they satisfy

$\nabla^{4} f_{n}=\lambda_{n} g_{n}, \quad \nabla^{2} g_{n}=-f_{n}, \quad \lambda_{n}=$ const.

These are precisely Eqs. (3) and (4) with $\epsilon=0$. Thus $f_{0}$ and $g_{0}$ are the solutions $w$ and $\theta$ of the Rayleigh stability problem, and $\left(\lambda_{0} / \alpha^{2}\right)=R_{0}$ is the critical Rayleigh number. When $\alpha=3.117$, we will have $R_{0} \approx 1708$. The $f_{n}$ and $g_{n}(n>0)$ are the higher modes mentioned by Pellew and Southwell. These functions are even or odd with respect to $z$ as $n$ is an even or odd integer. They may be computed explicitly in terms of trigonometric functions of complex argument, and depend on $\alpha$ as well as on $z$.

To establish the orthogonality of these functions, multiply the first of Eqs. (10) by $f_{m}$ and the second by $g_{m}$, and integrate from $z=-\frac{1}{2}$ to $z=+\frac{1}{2}$. After some manipulation we obtain

$$
\left(\lambda_{n}-\lambda_{m}\right) \int_{-\frac{1}{2}}^{+\frac{1}{2}} f_{n} g_{m} d z=0,
$$

and it is consistent to write

$$
\int_{-\frac{1}{3}}^{+\frac{1}{3}} f_{n} g_{m} d z=\delta_{n m}
$$

Then if the functions are complete, (8) will converge, and we have

$$
b_{n}=\int_{-\frac{1}{2}}^{+\frac{1}{1}} w g_{n} d z, \quad d_{n}=\int_{-\frac{1}{2}}^{+\frac{1}{2}} \theta f_{n} d z
$$


Conversely, it is not difficult to show that the Eqs. (8) converge in the mean, given (13). In fact, Eqs. (9) and (10) define a self-adjoint system, and the well-known theorems for Sturm-Liouville equations apply with only slight modifications. ${ }^{10}$ For problems related to the Rayleigh stability problem, these functions are often more useful than the simpler functions described by Gallagher and Mercer, ${ }^{6}$ and by Reid and Harris. ${ }^{11}$

To proceed, we multiply Eq. (3) by $f_{n}$ and Eq. (4) by $g_{n}$, and integrate from $z=-\frac{1}{2}$ to $z=+\frac{1}{2}$. With (7), (9), (10), and (13) the equations reduce to an infinite set of linear algebraic equations in the $b_{n}$ and $d_{n}$. The $d_{n}$ are easily eliminated, yielding

$$
\sum_{m=0}^{\infty}\left[\left(\lambda_{n}-\alpha^{2} R\right) \delta_{n m}+i E_{n m}+G_{n m}\right] b_{m}=0,
$$

where

$$
E_{n m}=\alpha \epsilon\left(\sigma D_{n m} \lambda_{m}-B_{n m}\right), \quad G_{n m}=\alpha^{2} \epsilon^{2} \sigma \sum_{k} D_{n k} B_{k m},
$$

and

$$
\begin{aligned}
& B_{n m}=\int_{-\frac{1}{2}}^{+\frac{1}{2}}(z-c) f_{n} \nabla^{2} f_{m} d z \\
& D_{n m}=\int_{-\frac{1}{2}}^{+\frac{1}{2}}(z-c) g_{n} g_{m} d z .
\end{aligned}
$$

Here $R$ is the unknown eigenvalue, and the $\lambda_{n}$ are the constants defined in (10). The functions $f_{n}$ and $g_{n}$ depend on $\alpha$ as well as on $z$, and thus the $B_{n m}$ and $D_{n m}$ may be computed given $\alpha$ and $c$. The eigenvalue $R$ is determined by requiring that the determinant of the coefficients $b_{m}$ in (14) be zero.

The determinant may be normalized by dividing each row by $\lambda^{1 / 6}$ and each column by $\lambda_{m}^{5 / 6}$. The diagonal term involving $R$ then has the form [ 1 $\left.\left(\alpha^{2} R / \lambda_{n}\right)\right]$, which approaches unity as $1+O\left(n^{-6}\right)$, for $n$ large. All other terms in the normalized determinant approach zero at least as fast as $1 / n$ or $1 / m$. Thus it is possible to truncate the infinite determinant to one of size $N \times N$, where $N$ is large, in evaluating the lowest eigenvalue, $R$.

This choice of eigenfunctions, Eqs. (10), leads to several simplifications in the calculation. Only for this choice was it possible to eliminate the $d_{n}$, as we did in deriving (14). This reduced the size of the determinant by one-half and the computing time by a factor of eight. In addition, the fact that

${ }^{10} \mathrm{H}$. Marganau and G. M. Murphy, The Mathematics of Physics and Chemistry (D. Van Nostrand Company, Inc., New York, 1956), 2nd ed., p. 277.

${ }^{11}$ W. H. Reid and D. L. Harris, Astrophys. J. Suppl. Ser. 3,429 (1958). the eigenvalue $R$ is only important in the first few diagonal terms makes it possible to obtain $R$ by a straightforward iteration procedure. Finally, this choice clarifies the nature of the solution for $\epsilon$ small, as we now demonstrate.

\section{SMALL REYNOLDS NUMBERS}

For $\epsilon$ arbitrarily small, we anticipate that $R$ will be expressible as a power series,

$$
R=R^{(0)}+\epsilon R^{(1)}+\epsilon^{2} R^{(2)}+\cdots .
$$

However, the determinant of the coefficients in (14) is complex, and so $R$ will be complex, for an arbitrary choice of $\sigma, \alpha, \epsilon$, and $c$. In order that the result be physically meaningful, $R$ must be real, which is sufficient to determine $c$. Thus we require, in addition to (16),

$$
c=c^{(0)}+\epsilon c^{(1)}+\epsilon^{2} c^{(2)}+\cdots .
$$

If we were also to require that $R$ be a minimum with respect to $\alpha$, then $\alpha$ would be a dependent variable, along with $R$ and $c$. For the present, however, $R^{(n)}$ and $c^{(n)}$ will be functions of $\sigma$ and $\alpha$, which we regard as independent variables, in addition to $\epsilon$.

The quantity $c$ is the phase speed of the disturbance relative to the basic velocity $\Delta u$. Therefore, we do not expect a singular limit in (17) as $\epsilon \rightarrow 0$. In fact, by cross multiplying (3) with the complex conjugate of (4), and integrating from $z=-\frac{1}{2}$ to $z=+\frac{1}{2}$, we may show that

$$
-\frac{1}{2}<c<+\frac{1}{2} \text {. }
$$

This states that the velocity of the disturbance may not be greater than that of either boundary and serves to justify (17).

We then substitute (15), (16), and (17) into (14), and set the determinant of the coefficients equal to zero to each order in $\epsilon$. The leading term is simply

$$
\prod_{n=0}^{\infty}\left[\lambda_{n}-\alpha^{2} R^{(0)}\right]=0,
$$

which is satisfied if $R^{(0)}$ in (16) is equal to $R_{0} \equiv \lambda_{0} / \alpha^{2}$, where $\lambda_{0}$ is the lowest of the set $\lambda_{n}$ in (10). This insures that the solutions of (3) and (4) will reduce asymptotically to solutions of the Rayleigh stability problem as $\epsilon \rightarrow 0$.

The terms proportional to $\epsilon$ give

$$
-\alpha^{2} R^{(1)} \epsilon+i E_{00}=0 .
$$

And since $f_{0}$ and $g_{0}$ are even functions of $z, E_{00}$ will be equal to $\epsilon c^{(0)}$ times a negative number, to this 
order in $\epsilon$. Thus the real part of (20) implies $R^{(1)}=0$, and the imaginary part implies $c^{(0)}=0$.

In order to evaluate the terms in higher powers of $\epsilon$, we must recall that the $f_{n}$ and $g_{n}$ are even or odd functions of $z$ as $n$ is even or odd. $B_{n m}$ and $D_{n m}$ will therefore be proportional to $c$ if $n+m$ is even and will not involve $c$ if $n+m$ is odd. Thus the imaginary terms in $\epsilon^{2}$ give $E_{00}=0$, which is now proportional to $\epsilon^{2} c^{(1)}$, and implies $c^{(1)}=0$.

The real terms proportional to $\epsilon^{2}$ give

$$
\begin{aligned}
-\alpha^{2} R^{(2)} \epsilon^{2} & +G_{00} \\
& +\sum_{m \text { odd }}\left(\lambda_{m}-\lambda_{0}\right)^{-1}\left(E_{0 m} E_{m 0}\right)=0
\end{aligned}
$$

which is an explicit expression for $R^{(2)}$ in terms of $\sigma$ and $\alpha$. This may be rewritten

$$
R^{(2)}=\sum_{m \text { odd }} \frac{\left[\sigma^{2} \lambda_{0} \lambda_{m} D_{0 m}^{2}-\sigma \lambda_{0} D_{0 m}\left(B_{0 m}+B_{m 0}\right)+B_{0 m} B_{m 0}\right]}{\left[\lambda_{m}-\lambda_{0}\right]},
$$

where $\lambda_{0}, \lambda_{m}, D_{0 m}, B_{0 m}$, etc, depend only on $\alpha$, for $m$ odd.

In this way one may continue the expansion to higher orders in $\epsilon$. It is possible to prove by induction that $c^{(n)}=0($ all $n)$, and that $R^{(n)}=0(n$ odd $)$. Thus for $\epsilon$ small enough, only stationary disturbances are allowed; there are no neutrally stable solutions in the limit $\epsilon \rightarrow 0$ for which $c \neq 0$.

We now have $R$ as a function of $\sigma, \alpha$, and $\epsilon$, for $\epsilon$ arbitrarily small. We may also minimize $R$ with respect to $\alpha$, in which case $\alpha$ becomes a function of the two other independent variables, $\sigma$ and $\epsilon$. Therefore, let

$$
\alpha=\alpha^{(0)}+\epsilon^{2} \alpha^{(2)}+\epsilon^{4} \alpha^{(4)}+\cdots,
$$

and expand each $R^{(n)}(\sigma, \alpha)$ in (16) as a power series in $\epsilon^{2}$, using (23). Choose the $\alpha^{(n)}(\sigma)$ such that $R$ is minimized to each order in $\epsilon^{2}$, whence

$$
\begin{gathered}
\alpha^{(0)}=\alpha_{0} \approx 3.117, \\
\alpha^{(2)}=-\left(\partial R^{(2)} / \partial \alpha\right)\left(\partial^{2} R^{(0)} / \partial \alpha^{2}\right)^{-1}, \\
R=R_{0}+\epsilon^{2} R^{(2)}+\epsilon^{4} \\
\cdot\left[R^{(4)}-\frac{1}{2}\left(\partial R^{(2)} / \partial \alpha\right)^{2}\left(\partial^{2} R^{(0)} / \partial \alpha^{2}\right)^{-1}\right]+\cdots,
\end{gathered}
$$

where the functions $R^{(n)}$ are evaluated at $\alpha=3.117$.

We have calculated these terms explicitly to order $\epsilon^{2}$. The minimum Rayleigh number at neutral stability is

$$
\begin{aligned}
R= & 1707.76 \\
& +\epsilon^{2}\left[0.5598 \sigma^{2}+0.1270 \sigma+0.06451\right],
\end{aligned}
$$

and the critical wavenumber is $\alpha=3.117-\epsilon^{2} 10^{-4}\left[2.325 \sigma^{2}+1.503 \sigma+1.155\right]$.

For the cases studied by Deardorff and by Gallagher and Mercer, these formulas are useful for $\epsilon<100$. Their main advantage is that the dependence on $\epsilon$ and $\sigma$ appears explicitly. For all $\sigma$, the minimum Rayleigh number at neutral stability increases, and the critical wavenumber decreases with $\epsilon$, for $\epsilon$ small. Equation (26) constitutes a proof of stability at $R<R_{0}$, for $\epsilon$ arbitrarily small.

\section{THE GENERAL NUMERICAL SOLUTION}

For finite $\epsilon$, one may solve the infinite determinant in (14) numerically. Truncate the determinant to one of size $N \times N$; compute the lowest eigenvalue $R$ for several $N$, and take the limit as $N \rightarrow \infty$. To obtain the lowest eigenvalue of the truncated determinant, substitute a trial value into each term except the lowest diagonal; choose $R$ in the lowest diagonal so that the determinant will be zero, and use this $R$ as the next trial value in the iteration.

If $c$ is zero, eigenvalue $R$ will always be real. For $c$ different from zero, $R$ will be real only for certain discrete values of $c$, provided $\epsilon$ is large enough and $c$ is less than one-half. These traveling disturbances were located graphically by computing complex $R$ at several arbitrary values of $c$ and then setting $\operatorname{Im}(R)=0$.

This method was used to obtain neutral stability curves for both stationary and traveling disturbances. ${ }^{7}$ Subsequently, the writer has checked several values reported by Deardorff and by Gallagher and Mercer and has found satisfactory agreement. For instance, Gallagher and Mercer report a neutrally stable traveling disturbance at $\sigma=1, \alpha=4$, $\epsilon=133$ and $R \approx 0.3004 \times 10^{5}$. Using a $20 \times 20$ matrix, at the same values of $\sigma, \alpha$, and $\epsilon$, we find a neutral disturbance at $c=0.0885$ and $R \approx$ $0.3080 \times 10^{5}$. (Gallagher and Mercer do not report the phase speed of the disturbance.)

Below we consider several questions raised by the work of Deardorff and Gallagher and Mercer. The first concerns the role of traveling disturbances. In Fig. 1 we give the Rayleigh number at neutral stability as a function of $\epsilon$, for $\sigma=10$ and $\alpha=3.0$. The first four stationary modes are shown as solid curves, and the lowest of these exists only for $\epsilon<150$. Thus one effect of increasing the transverse Reynolds number $\epsilon$ is to inhibit stationary disturbances. Where the first two stationary modes join, a pair of traveling disturbances appear, corresponding to the two solutions $c= \pm c(\epsilon)$. For 


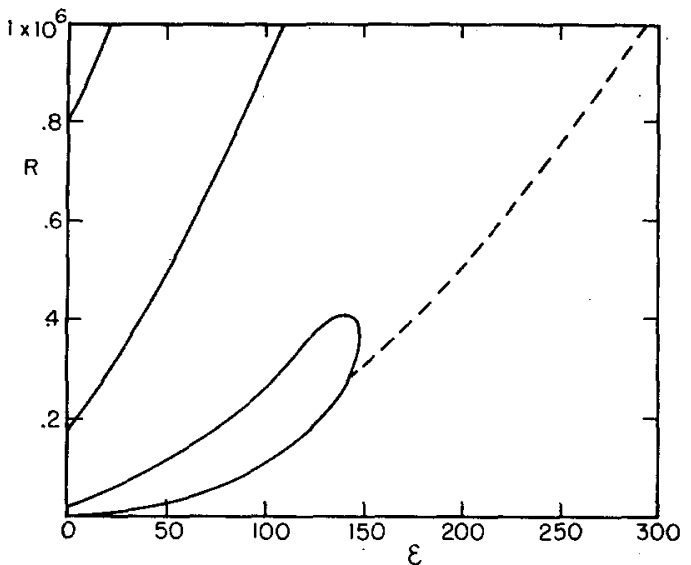

FIG. 1. Neutral stability curves: $R$ vs $\epsilon$, with $\sigma=10$ and $\alpha=3$. The solid curves are the first four stationary modes; the broken curve is a traveling mode. Similar curves calculated for $\alpha=3.3$ lie slightly above and to the left of these curves.

these traveling disturbances, the Rayleigh number at neutral stability continues to increase with $\epsilon$, as shown by the broken curve in Fig. 1. Thus the estimated curve in Fig. 6 of the paper by Gallagher and Mercer is qualitatively correct.

The phase speed of these traveling disturbances is given in Fig. 2 as a function of $\epsilon$. As $\epsilon \rightarrow \infty$, the phase speed may approach $c=\frac{1}{2}$, the speed of the upper boundary, although this cannot be inferred from the present data.

In Fig. 3 the Rayleigh number at neutral stability is plotted against $\alpha$, for $\sigma=10$ and $\epsilon=150$. The minimum value of $R$, at $\alpha \approx 1.5$, corresponds to the first stationary mode. Traveling disturbances only appear at larger Rayleigh numbers and at higher wavenumbers; the higher modes also appear only at larger Rayleigh numbers. It is possible that very different behavior might occur for different values of $\sigma$ and $\epsilon$, although Figs. 1-3 are typical of several other cases studied. This gives support

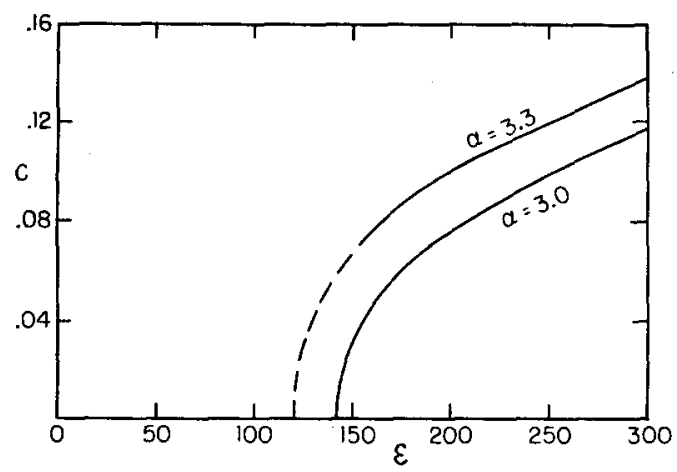

FIg. 2. Phase speed at neutral stability: $c$ vs $\epsilon$, with $\sigma=10$ and $\alpha=3.0,3.3$. The broken part of the curve was estimated visually. to the assumption of Gallagher and Mercer, that traveling disturbances and higher modes may be neglected in discussing the minima of the neutral stability curves.

We have also investigated the limit of large Prandtl numbers, which was not considered by Deardorff or by Gallagher and Mercer. For $\epsilon$ small, (26) indicates that the minimum critical Rayleigh number depends only on $\epsilon^{2}$ for $\sigma$ small, and on $(\sigma \epsilon)^{2}$ for $\sigma$ large. The same behavior occurs at finite $\epsilon$. For example, using a $20 \times 20$ matrix, with $\sigma \epsilon=$ $2000 / 3, \alpha=3.3$ and $c=0$, the following holds asymptotically for $\sigma$ large:

$$
R(\sigma) \sim 2.73 \times 10^{4}[1+(4.7 / \sigma)+\cdots] .
$$

The neutral stability curves approach a definite limit as $\sigma \rightarrow \infty$ with $\sigma \epsilon$ fixed. Moreover, we have

$$
\sigma \epsilon=\operatorname{Pe} \alpha_{x} / \alpha=(\Delta u h / \kappa)\left(\alpha_{x} / \alpha\right),
$$

where the quantity $\mathrm{Pe}$ is the Peclet number of the basic state, and plays the role of a Reynolds number in the heat equation, (4). Thus for $\sigma$ large, the mean shear is important only in the heat equation, while for $\sigma$ small, the mean shear is important only in the momentum equation.

In Fig. 4 we give some neutral stability curves for stationary disturbances as $\sigma \rightarrow \infty$. Clearly, the minimum of $R$ with respect to $\alpha$ is a monotonically increasing function of $\sigma \epsilon$. This is qualitatively the same as in Figs. 2-4 of the paper by Gallagher and Mercer, for which $\sigma=0,0.7$, and 6 , respectively. Traveling disturbances also exist as $\sigma \rightarrow \infty$ and seem to behave as in Figs. 1-3 of the present paper. Thus it appears that for all $\sigma$, the minimum Rayleigh

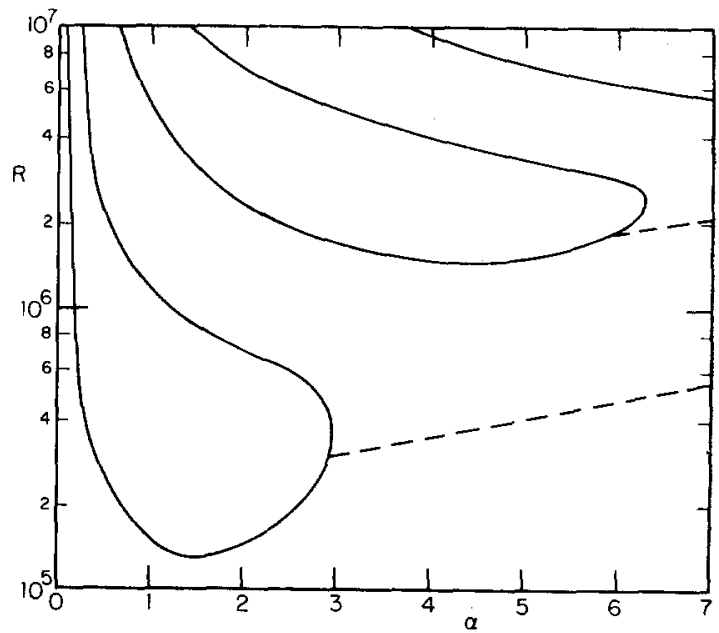

Fig. 3. Neutral stability curves: $R$ vs $\alpha$, with $\sigma=10$ and $\epsilon=150$. The solid curves are the first five stationary modes. The broken curves are traveling modes. 


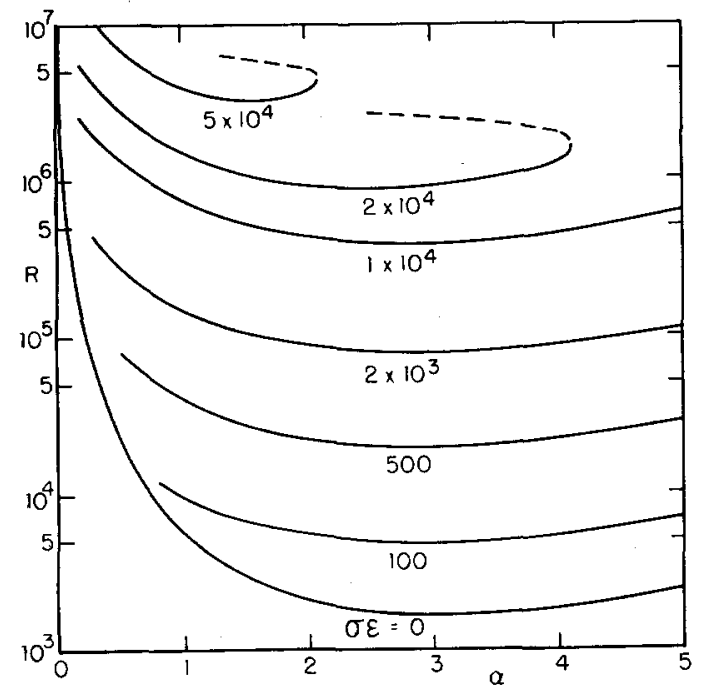

FIG. 4. Neutral stability curves for stationary disturbances: $R$ vs $\alpha$ for various $\sigma \epsilon$, with $\sigma \rightarrow \infty$ and $\epsilon \rightarrow 0$. The broken curves are the second stationary mode (estimated).

number at neutral stability increases monotonically with $\epsilon$ and corresponds to a stationary disturbance.

\section{LARGE REYNOLDS NUMBERS}

Consider next the limit as $\epsilon$, the Reynolds number for transverse disturbances, becomes infinite. As stated in the Introduction, our primary aim is to determine whether unstable disturbances exist for $R<1708$; therefore, we shall assume that $R, \sigma$, and $\alpha$ remain finite as $\epsilon \rightarrow \infty$ and shall treat $c$ as the complex eigenvalue. Let $c=c_{r}+i c_{i}$; then $c_{i}>0$ implies instability.

A heuristic treatment of the Orr-Sommerfeld equation at large Reynolds numbers is described by Lin, Sec. 8.5. ${ }^{12}$ Our treatment of Eqs. (3) and (4) as $\epsilon \rightarrow \infty$ is quite similar, and therefore, we only outline the procedure. A fundamental set of six asymptotic solutions may be obtained for $z-c$ finite by expanding in powers of $(\alpha \epsilon)^{-\frac{1}{2}}$. Four of these are asymptotically solutions of the OrrSommerfeld equation for plane Couette flow, Eq. (3) with right side equal to zero. The corrections due to thermal effects are of order $J$ or smaller, where

$$
J=\left[R /\left(\sigma \epsilon^{2}\right)\right] \rightarrow 0 \text { as } \epsilon \rightarrow \infty .
$$

The other two solutions are obtained by setting the right side of (4) equal to zero in the first approximation. These six asymptotic solutions are multivalued about the point $z=c$, although the basic equations are regular there. In other words, the

${ }^{12}$ C. C. Lin, The Theory of Hydrodynamic Stability (Cambridge University Press, Cambridge, England, 1955). asymptotic solutions are representations of different actual solutions of (3) and (4) in different sectors of the complex $z-c$ plane.

We may obtain a second representation of the six fundamental solutions by introducing the transformation

$$
\eta=(\alpha \epsilon)^{\frac{1}{3}}(z-c),
$$

and expanding in powers of $(\alpha \epsilon)^{-t}$. These solutions are regular at $z=c$, and may be expressed in terms of solutions of Stokes' equation,

$$
y^{\prime \prime}+x y=0, \text { i.e., } \quad y=x^{\frac{1}{2}} H_{\frac{1}{3}}^{(1,2)}\left(\frac{2}{3} x^{\frac{3}{3}}\right) .
$$

Again the effect of coupling between Eqs. (3) and (4) is of order $J$. This representation of the solutions is presumably only valid for $\eta$ finite, that is, in a region of the $z$ plane about $z=c$ of radius $(\alpha \epsilon)^{-\frac{1}{2}}$. However, we may compare the asymptotic expansions of these solutions for large $\eta$ with the asymptotic solutions described in the previous paragraph. We find a unique correspondence of solutions for

$$
-(7 \pi / 6)<\arg (\eta)<(\pi / 6),
$$

which is the same condition as in the Couette flow stability problem. ${ }^{13-15}$ For slightly damped, neutral or unstable disturbances, this region includes the boundaries at $z= \pm \frac{1}{2}$.

A linear combination of the six fundamental solutions must satisfy the boundary conditions (7). This leads to a transcendental equation for the eigenvalue, $c$, in terms of $\sigma, \alpha, \epsilon$, and $R$. This is of the form

$$
F(c, \alpha, \epsilon) G(c, \alpha, \sigma \epsilon)+O(J)=0,
$$

where $F$ and $G$ are functions of order unity as $\alpha \epsilon \rightarrow \infty$. The solutions of $F(c, \alpha, \epsilon)=0$ are eigenvalues of the Couette flow stability problem, and correspond to damped disturbances as $\alpha \epsilon \rightarrow \infty$. For the related solutions of (31), one finds

$$
-(\alpha \epsilon)^{\frac{3}{5}} c_{i} \geq 1.0626 P_{1}(c, \alpha, \epsilon)+O(\sigma J),
$$

where $P_{1}$ is a power series in $(\alpha \epsilon)^{-\frac{1}{3}}$, starting with unity, and we have used the numerical results of Zondek and Thomas. ${ }^{14}$ Thus for $\epsilon$ sufficiently large ( $J$ sufficiently small), these modes will decay exponentially in time.

Similarly, the solutions of $G(c, \alpha, \sigma \epsilon)=0$ are eigenvalues of the equation

$\left[\nabla^{2}-i \sigma \alpha \epsilon(z-c)\right] \theta=0, \quad \theta=0$ at $z= \pm \frac{1}{2}$.

${ }_{13}$ L. Hopf, Ann. Physik 44, 1 (1914).

14 B. Zondek and L. H. Thomas, Phys. Rev. 90, 738 (1953).

15 W. Wasow, J. Res. Natl. Bur. Std. (U. S.) 51, 195 (1953). 
For these disturbances, one finds

$$
-(\sigma \alpha \epsilon)^{\frac{1}{3}} c_{i} \geq P_{2}(c, \alpha, \sigma \epsilon)+O(J / \sigma),
$$

where $P_{2}$ is a power series in $(\sigma \alpha \epsilon)^{-1}$, starting with a positive constant. One may also prove stability simply by multiplying (33) by $\theta^{*}$ and integrating from $z=-\frac{1}{2}$ to $z=+\frac{1}{2}$. Thus the sixth-order system (3) and (4) is stable as $\epsilon \rightarrow \infty$, for $\sigma, \alpha$, and $R$ finite. If $\alpha$ is unbounded, the results (32) and (34) may not hold, but we may prove stability following Zondek and Thomas, Sec. XII.

There are two other limiting cases that are tractable in this way. The first is $\alpha \epsilon \rightarrow \infty, R$ finite, $\sigma=0$ (i.e., $\sigma \alpha \epsilon \rightarrow 0$ ). Here, the only eigenvalues are those of the Couette flow stability problem, for which the result (32) still holds. The other case is $\sigma \alpha \epsilon \rightarrow \infty, R$ finite, $\sigma=\infty$ (i.e., $\alpha \epsilon \rightarrow 0$ ), in which case the only eigenvalues are those of Eq. (33), for which (34) holds. In both cases, the flow is stable for $R$ finite.

This completes the analysis of the physically interesting case $R<R_{0}$, as $\epsilon \rightarrow \infty$. It would be mathematically interesting to consider the case $J$ fixed as $\epsilon \rightarrow \infty$ (i.e., $R \rightarrow \infty$ ). If $\alpha \epsilon$ and $\sigma \alpha \epsilon$ are both large, two of the asymptotic solutions will satisfy the "inviscid" equation

$$
(z-c)^{2} \nabla^{2} w-J w=0,
$$

in the first approximation. This equation is singular at $z=c$, and so we must look at the full equations in a neighborhood of the critical point in order to determine the complete behavior of the solutions of (35). Unfortunately, this leads to a rather complicated sixth-order equation in the variable $\eta=$ $(\alpha \epsilon)^{t}(z-c)$, involving the constants $\sigma$ and $J$. We have not solved this equation, but we make the simplest possible hypothesis, namely, that the solutions of (35) are valid on the real $z$ axis, except possibly in an inner friction layer.

We should then expect a set of eigenvalues related to those of the inviscid equation (35), with boundary conditions $w=0$ at $z= \pm \frac{1}{2}$. 'These have been computed by Kuo, ${ }^{4}$ who considered Eq. (35) in the region $|z| \leq 1$, with $-J$ replaced by $\bar{J}$, which Kuo calls the modified Richardson number. (Our $c_{r}$ corresponds to his $\frac{1}{2} q_{r}$, and our $\alpha$ to his $2 \alpha$.) If $J$ is plotted against $\alpha$, the neutral stability curve for stationary disturbances has zero slope at $J=0.75$ and $\alpha=0$. It then increases monotonically with $\alpha$, and passes through the point $J=2.0$, $\alpha=2.3994$, where a pair of neutrally stable traveling disturbances appear. These occur along the line $J=2.0, \alpha>2.3994$ (Kuo, Fig. 4). This behavior is qualitatively the same as in Fig. 3 of our paper, except near $\alpha=0$. In fact, for the lowest traveling mode in Fig. 3, $J$ is about $2.0 \pm 50 \%$. The results in Fig. 1, and an isolated point for $\sigma=1, \alpha=4$, $\epsilon=133, R \approx 0.3080 \times 10^{5}$ also agree roughly with the inviscid result. Thus it appears likely that the neutral stability curves obtained numerically for $\alpha \epsilon$, and $\sigma \alpha \epsilon$ large are closely related to the solutions of (35). If this is so, we should expect $R$ at neutral stability to increase roughly as $\sigma \epsilon^{2}$, for $\epsilon$ large enough, with $\alpha$ and $\sigma$ fixed.

The phase speed for neutrally stable solutions of (35) is given in Fig. 2(a) of Kuo's paper. Thus as $\epsilon \rightarrow \infty$, we should expect $c$ to be a unique function of $\alpha$, with $c=0$ at $\alpha \leq 2.3994$ and $c \rightarrow \frac{1}{2}$ as $\alpha \rightarrow \infty$. For $\alpha=3.0$, we should have $c \approx 0.16$, and for $\alpha=3.3, c \approx 0.20$, which is consistent with Fig. 2 of our paper.

As mentioned above, Eq. (35) does not apply if $\alpha \epsilon$ or $\sigma \alpha \epsilon$ are small. Thus the lowest curve in Fig. 3 of our paper does not agree with Kuo's neutral stability curve as $\alpha \rightarrow 0$. Similarly, the results in Fig. 4 of our paper, for which $\epsilon$ is vanishingly small ( $\sigma \rightarrow \infty$ with $\sigma \epsilon$ fixed), cannot be compared with Kuo's solutions. The same is true for Fig. 2 of Gallagher and Mercer, for which $\sigma=0$. On the other hand, for $R$ finite as $\epsilon \rightarrow \infty$, the flow is stable for all $\alpha$ and $\sigma$ as was shown in the first part of this section.

\section{COMPARISON WITH EXPERIMENT}

From the preceding analysis, and from the work of Deardorff and of Gallagher and Mercer, convection with shear appears to be stable at $R<1708$. We have proved this conclusion only for $\epsilon$ infinitely small and infinitely large. For finite $\epsilon$, we must assume that the cases investigated numerically are typical of the flow at all values of $\sigma, \alpha, \epsilon$, and $c$. Therefore, at $R=1708$ we should expect convection to appear as longitudinal rolls, for which $\epsilon=0$ irrespective of the rate of shear.

Benard and Avsec, ${ }^{1}$ Chandra, ${ }^{2}$ and Brunt ${ }^{3}$ describe experiments on air in which shear was produced in a straight channel by moving one of the horizontal surfaces bounding the fluid. Cigarette smoke was used to mark the fluid and to detect the onset of convection. They report no cases of instability at values of the Rayleigh number less than 1708. Recently, the author ${ }^{7,16}$ measured heat flux and momentum flux in liquids in an annular channel. It was not possible to observe the flow

\footnotetext{
${ }^{16}$ A. P. Ingersoll, J. Fluid Mech. (to be published).
} 
in these experiments. In Fig. 5 we give the Nusselt number (dimensionless heat flux) as a function of Rayleigh number, for several values of $R e$, the Reynolds number of the basic state. The curves are almost identical, indicating that the shear has little effect on the onset of convection, or on the heat flux for $\left(R / R_{0}\right) \leq 3$. These data are in accord with the theoretical results presented in this paper. The effect of convection on the momentum flux was too small to be measured accurately at these Rayleigh numbers.

It is more difficult to explain the form of the convective cells observed in the experiments on air. Transverse rolls appear at small rates of shear, whereas longitudinal rolls only occur above a certain critical rate of shear. However, the experiments are not inconsistent with the results of the stability analysis presented here. Invariably, transverse rolls only appear at Rayleigh numbers considerably above the critical values predicted in this paper. For instance, Brunt reports that at $\Delta T=$ $40^{\circ} \mathrm{C}$ and $h=1.0 \mathrm{~cm}$, the transition from transverse to longitudinal rolls occurs at $\Delta u=0.5 \mathrm{~cm} / \mathrm{sec}$. For this rate of shear, the critical Rayleigh number for transverse rolls in air is about 1712.4, from (26). However, Brunt's observations are for Rayleigh numbers greater than 4000. At these Rayleigh numbers, the difference in the stability with respect to longitudinal and transverse rolls is probably negligible compared to finite amplitude effects, which we have not considered here. Thus there is no need to reject either the theory or the experiments; further research is necessary before a direct comparison can be made. It is consistent that as $R \rightarrow 1708$ from above, the maximum Reynolds

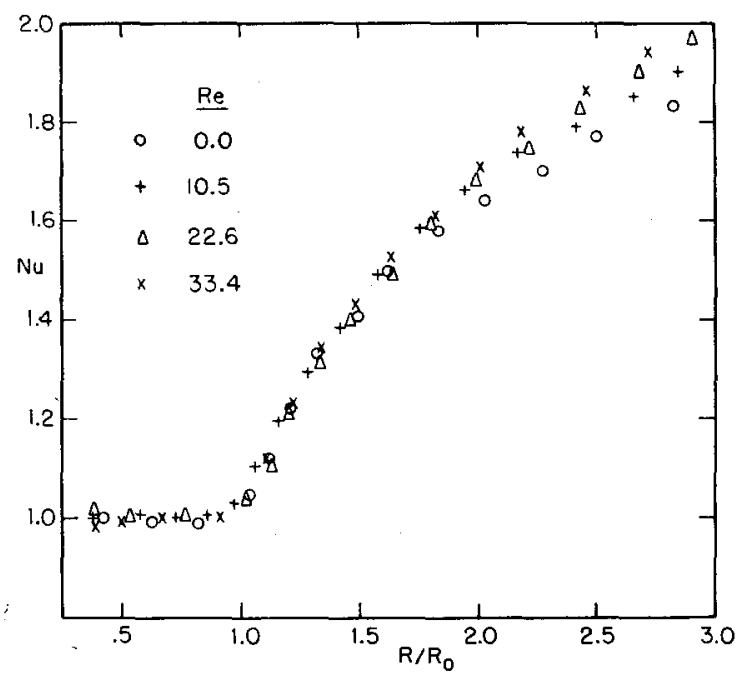

Frg. 5. Heat flux measurements: $\mathrm{Nu}$ vs $R / R_{0}$ for various Re, with $\sigma=9.35$. Here $R_{0}=1708$ is the critical Rayleigh number, $R e$ is the Reynolds number at the outer radius of the annular channel, and $\mathrm{Nu}$ is the Nusselt number.

number at which transverse rolls are observed approaches zero. Presumably for $\Delta u=0.5 \mathrm{~cm} / \mathrm{sec}$ and $1708<R<1712$, only longitudinal rolls would have been observed in Brunt's experiment.

\section{ACKNOWLEDGMENTS}

Part of this work was included in a doctoral thesis submitted to the department of physics at Harvard University. Professor Richard Goody provided essential guidance during the period of this research, and his help is gratefully acknowledged.

Financial support was provided by the National Science Foundation, under Grant G 24903 awarded to Harvard University. 